\title{
MULTIPLICATION THEOREMS ON STRONGLY SUMMABLE SERIES
}

\section{BABBAN PRASAD MISHRA ${ }^{1}$}

\section{Introduction.}

1.1. Let $\left\{\lambda_{n}\right\}$ be an arbitrary increasing sequence of positive numbers, such that

$$
0<\lambda_{0}<\lambda_{1}<\lambda_{2}<\cdots<\lambda_{n-1}<\lambda_{n} \rightarrow \infty \text {, as } n \rightarrow \infty,
$$

and $\sum_{n=0}^{\infty} a_{n}$ a given series.

We write

$$
A_{n}=a_{0}+a_{1}+a_{2}+\cdots+a_{n-1}+a_{n}, \quad A_{\lambda}(\omega)=0, \text { for } \omega \leqq h,
$$

where $h$ is a convenient positive number.

If $\omega>0, \lambda_{n}<\omega<\lambda_{n+1}$ then

$$
A_{\lambda}(\omega)=A_{n}=\sum_{v=0}^{n} a_{v}=\sum_{\lambda_{v}<\omega} a_{v}
$$

and for $k>0$

$$
\begin{aligned}
A_{\lambda}^{k}(\omega) & =\sum_{\lambda_{n}<\omega}\left(\omega-\lambda_{n}\right)^{k} a_{n} \\
& =k \int_{0}^{\omega}(\omega-t)^{k-1} A_{\lambda}(t) d t=\int_{0}^{\omega}(\omega-t)^{k} d A_{\lambda}(t) .
\end{aligned}
$$

We define $A_{\lambda}^{0}(\omega)=A_{\lambda}(\omega)$. We also define

$$
\begin{aligned}
\bar{A}_{\lambda}^{k}(\omega) & =\sum_{\lambda_{n}<\omega}\left(\omega-\lambda_{n}\right)^{k-1} \lambda_{n} a_{n} & & (k>0) \\
& =-\int_{0}^{\omega} A_{\lambda}(t) \frac{d}{d t}\left[(\omega-t)^{k-1} t\right] d t & & (k>1) \\
& =\int_{0}^{\omega}(\omega-t)^{k-1} t d A_{\lambda}(t) & & (k \geqq 1) .
\end{aligned}
$$

We have

Received by the editors October 12, 1964 and, in revised form, August 30, 1965 and February 8, 1966.

1 This research has been supported by a Junior Research Fellowship of The Council of Scientific and Industrial Research, India. 


$$
\frac{d}{d \omega}\left(\frac{A_{\lambda}^{k}(\omega)}{\omega^{k}}\right)=\frac{k}{\omega^{k+1}} \bar{A}_{\lambda}^{k}(\omega) .
$$

We use $B_{\mu}^{k}(\omega), \bar{B}_{\mu}^{k}(\omega)$ and $C_{\nu}^{k}(\omega), \bar{C}_{\nu}^{k}(\omega)$ for similar expressions involving $\sum_{n=0}^{\infty} b_{n}$ and $\sum_{n=0}^{\infty} c_{n}$ respectively.

1.2. If we associate summability by Riesz means of type $\lambda$ with the series $\sum_{n=0}^{\infty} a_{n}$ and type $\mu$ with $\sum_{n=0}^{\infty} b_{n}$, we may form the sequence of numbers $\nu_{n}$, which are numbers $\lambda_{p}+\mu_{q}$ arranged in increasing order of magnitude, and associate summability by Riesz means of type $\nu$ with the series $\sum_{n=0}^{\infty} c_{n}$, where

$$
c_{n}=\sum_{\lambda_{p}+\mu_{q}=\nu_{n}} a_{p} b_{q} \text {. }
$$

Then we call $\sum_{n=0}^{\infty} c_{n}$ the Dirichlet product of $\sum_{n=0}^{\infty} a_{n}$ and $\sum_{n=0}^{\infty} b_{n}$. If $\lambda_{n}=\mu_{n}=n$, then the rule reduces to Cauchy's.

2. Definitions. The series $\sum_{n=0}^{\infty} a_{n}$ is said to be summable $(R, \lambda, k)$, where $k \geqq 0$, to the sum $s$ if

$$
\lim _{\omega \rightarrow \infty} A_{\lambda}^{k}(\omega) / \omega^{k}=s \quad \text { (cf. [3]). }
$$

If, in addition,

$$
\int_{h}^{\omega}\left|u \frac{d}{d u}\left(\frac{A_{\lambda}^{k}(u)}{u^{k}}\right)\right|^{r} d u=o(\omega),
$$

as $\omega \rightarrow \infty$, then the series $\sum_{n=0}^{\infty} a_{n}$ is said to be summable $[R, \lambda, k, r]$ to the sum $s,\left(k>0, r \geqq 1, k>1 / r^{\prime}\right)$, where $r^{\prime}$ denotes the number conjugate to $r$, i.e. $r^{\prime}=r /(r-1)$ [5]. We define $r^{\prime}$ to be $\infty$ if $r=1$.

For the definition to be valid at all, the condition $k>1 / r^{\prime}$ is essential as pointed out by Boyd and Hyslop [2, pp. 94-95].

When $r=1,[R, \lambda, k]$ and $[R, \lambda, k, r]$ denote the same method. Now $[R, \lambda, 0]$ summability is equivalent to convergence and

$$
\int_{h}^{\boldsymbol{X}} x\left|d A_{\lambda}^{0}(x)\right|=o(X), \text { as } X \rightarrow \infty .
$$

The above condition is the same as

$$
\sum_{\lambda_{n}<X}\left|a_{n} \lambda_{n}\right|=o(X)
$$

[5]. We observe that on account of (1.1) the condition (2.1) is equivalent to 


$$
\int_{h}^{x}\left|\frac{\bar{A}_{\lambda}^{k}(u)}{u^{k}}\right|^{r} d u=o(X), \text { as } \quad X \rightarrow \infty .
$$

Again, since $h>0$ and $\bar{A}_{\lambda}^{k}(u)$ is integrable $(L)$ in the range $(h, X)$ for every finite $X>h$, the condition (2.2) is equivalent to

$$
\int_{h}^{X}\left|\bar{A}_{\lambda}^{k}(u)\right|^{r} d u=o\left(X^{k r+1}\right), \quad \text { as } \quad X \rightarrow \infty \quad[5] .
$$

The assertion that the series $\sum_{n=0}^{\infty} a_{n}$ is summable $|R, \lambda, 0|$ to $s$ means that $\sum_{n=0}^{\infty} a_{n}=s$ (in the usual sense) and $\sum_{n=0}^{\infty}\left|a_{n}\right|<\infty$.

It has been shown by Srivastava [5, p. 68, Theorem 9 and p. 61, Theorem 1] that, for $k \geqq 0$, summability $|R, \lambda, k|$ implies summability $[R, \lambda, k]$ and so also summability $(R, \lambda, k)$.

3. The following theorems are known.

Theorem 1. If $\sum_{n=0}^{\infty} a_{n}$ is summable $(R, \lambda, k)$ to sum $s, k \geqq 0$, and $\sum_{n=0}^{\infty} b_{n}$ is summable $(R, \mu, l)$ to sum $t$, then $\sum_{n=0}^{\infty} c_{n}$ is summable $(R, \nu, k+l+1)$ to sum st, $(l \geqq 0)$.

Theorem 2. If $\sum_{n=0}^{\infty} a_{n}$ is summable $[R, \lambda, k], k>0$, to sum $s$ and $\sum_{n=0}^{\infty} b_{n}$ is summable $(R, \mu, l)$ to sum $t$, then the series $\sum_{n=0}^{\infty} c_{n}$ is summable $(R, \nu, k+l)$ to sum st.

Theorem 3. If $\sum_{n=0}^{\infty} a_{n}$ is summable $[C, k]$, where $k>0$, to $s$ and $\sum_{n=0}^{\infty} b_{n}$ is summable $|C, 0|$ to $t$, then $\sum_{n=0}^{\infty}\left(a_{0} b_{n}+a_{1} b_{n-1}+\cdots\right.$ $\left.+a_{n} b_{0}\right)$ is summable $[C, k]$ to st.

Theorems 1 and 2 are due to Chandrasekharan and Minakshisundaram [3, p. 100, Corollary 3.91 and p. 106, Theorem 3.96]. Theorem 3 has recently been obtained by A. V. Boyd [1]. We obtain in Theorem A the analogue of Theorem 3 for the Dirichlet product. Theorem B is concerned with summability $[R, \lambda, k, r]$ instead of summability $[R, \lambda, k]$.

We shall prove the following theorems.

Theorem A. If $\sum_{n=0}^{\infty} a_{n}$ is summable $[R, \lambda, k]$, where $k>0$, to $s$ and $\sum_{n=0}^{\infty} b_{n}$ is summable $|R, \mu, 0|$ to $t$, then $\sum_{n=0}^{\infty} c_{n}$ is summable $[R, \nu, k]$ to sum st.

THEOREM B. If $\sum_{n=0}^{\infty} a_{n}$ is summable $[R, \lambda, k, r]$, where $k>1 / r^{\prime}$ and $r>1$, to $s$ and $\sum_{n=0}^{\infty} b_{n}$ is summable $|R, \mu, 0|$ to $t$, then $\sum_{n=0}^{\infty} c_{n}$ is summable $[R, \nu, k, r]$ to st.

We observe that Theorem $\mathrm{B}$ reduces to Theorem $\mathrm{A}$ when $r=1$. It 
may be mentioned that Theorem A of the present paper includes as a particular case a theorem of Boyd [1] for strong Cesàro summability on account of equivalence of summabilities $[R, n, k]$ and $[C, k][2]$.

I am grateful to Dr. (Mrs.) Sulaxana K. Gupta and Dr. Pramila Srivastava for their kind suggestions during the preparation of this paper. I am also indebted to the referee for valuable suggestions according to which I have modified my paper.

4. We require the following lemma.

Lemma. Suppose that $1 \leqq r<\infty$ and $k>0$. Then, if the series $\sum_{n=0}^{\infty} a_{n}$ is summable $(R, \lambda, \alpha)$ for some $\alpha>0$ to the sum s, and

$$
\int_{h}^{x}\left|\bar{A}_{\lambda}^{k}(u)\right|^{r} d u=o\left(X^{k r+1}\right), \text { as } X \rightarrow \infty,
$$

then it is summable $[R, \lambda, k, r]$ to the sum $s$.

This result is analogous to Flett's Theorem 7 [4] on strong Cesàro summability. The lemma follows by combining Corollaries 1 and 2 to Theorem 8 of Srivastava [5, p. 66].

5. It is convenient first to prove Theorem $B$.

Under the hypothesis of the theorem, $\sum_{n=0}^{\infty} a_{n}$ is summable $(R, \lambda, k)$ to the sum $s$ and $\sum_{n=0}^{\infty} b_{n}$ is summable $(R, \mu, 0)$ to the sum $t$. Applying Theorem 1, we deduce summability $(R, \nu, k+1)$ of $\sum_{n=0}^{\infty} c_{n}$ to the sum st. Hence by the lemma it is sufficient to prove that

$$
\int_{h}^{x}\left|\bar{C}_{\nu}^{k}(\omega)\right|^{r} d \omega=o\left(X^{k r+1}\right), \text { as } X \rightarrow \infty .
$$

For $\omega \neq \lambda_{p}+\mu_{q}$,

$$
\begin{aligned}
\bar{C}_{\nu}^{k}(\omega)= & \sum_{\lambda_{p}+\mu_{q}<\omega}\left(\omega-\lambda_{p}-\mu_{q}\right)^{k-1}\left(\lambda_{p}+\mu_{q}\right) a_{p} b_{q} \\
= & \sum_{\mu_{q}<\omega} \mu_{q} b_{q} \sum_{\lambda_{p}+\mu q<\omega}\left(\omega-\lambda_{p}-\mu_{q}\right)^{k-1} a_{p} \\
& +\sum_{\mu_{q}<\omega} b_{q} \sum_{\lambda_{p}+\mu_{q}<\omega}\left(\omega-\lambda_{p}-\mu_{q}\right)^{k-1} \lambda_{p} a_{p} \\
= & \sum_{\mu_{q}<\omega} \mu_{q} b_{q} \cdot \frac{1}{\left(\omega-\mu_{q}\right)} A_{\lambda}^{k}\left(\omega-\mu_{q}\right) \\
& +\sum_{\mu_{q}<\omega} \mu_{q} b_{q} \cdot \frac{1}{\left(\omega-\mu_{q}\right)} \bar{A}_{\lambda}^{k}\left(\omega-\mu_{q}\right)+\sum_{\mu_{q}<\omega} b_{q} \bar{A}_{\lambda}^{k}\left(\omega-\mu_{q}\right) \\
= & P_{1}(\omega)+P_{2}(\omega)+P_{3}(\omega),
\end{aligned}
$$


say. Hence, by Minkowski's inequality, it is enough to prove that, if $P(\omega)$ is any one of $P_{1}(\omega), P_{2}(\omega), P_{3}(\omega)$, then

$$
\int_{h}^{x}|P(\omega)|^{r} d \omega=o\left(X^{k+1}\right), \text { as } X \rightarrow \infty .
$$

We observe that

$$
\left|P_{3}(\omega)\right|^{r}=\left|\sum_{\mu_{q}<\omega}\left\{\left(b_{q}\right)^{1 / r} \bar{A}_{\lambda}^{k}\left(\omega-\mu_{q}\right)\right\} \times\left\{\left(b_{q}\right)^{1 / r^{\prime}}\right\}\right|^{r} .
$$

Applying Hölder's inequality for sums with indices $r$ and $r^{\prime}$, we have

(5.3) $\left|P_{3}(\omega)\right|^{r} \leqq\left\{\sum_{\mu_{q}<\omega}\left|b_{q}\right|\left|\bar{A}_{\lambda}^{k}\left(\omega-\mu_{q}\right)\right|^{r}\right\}\left\{\sum_{\mu_{q}<\omega}\left|b_{q}\right|\right\}^{r / r^{\prime}}$.

We have, since $\sum_{n=0}^{\infty} b_{n}$ is summable $|R, \mu, 0|$,

$$
\left|P_{3}(\omega)\right|^{r} \leqq M \sum_{\mu q<\omega}\left|b_{q}\right|\left|\bar{A}_{\lambda}^{k}\left(\omega-\mu_{q}\right)\right|^{r},
$$

where $M$ is a constant.

Hence

$$
\int_{h}^{x}\left|P_{3}(\omega)\right|^{r} d \omega \leqq M \int_{h}^{x} \sum_{\mu_{q}<\omega}\left|b_{q}\right|\left|\bar{A}_{\lambda}^{k}\left(\omega-\mu_{q}\right)\right|^{r} d \omega .
$$

Interchanging the order of integration and summation, we get

$$
\begin{aligned}
\int_{h}^{X}\left|P_{3}(\omega)\right|^{r} d \omega & \leqq M \sum_{\mu_{q}<X}\left|b_{q}\right| \int_{\mu_{q}}^{X}\left|\bar{A}_{\lambda}^{k}\left(\omega-\mu_{q}\right)\right|^{r} d \omega \\
& =M \sum_{\mu_{q}<X}\left|b_{q}\right| o\left(X^{k r+1}\right) \\
& =o\left(X^{k r+1}\right),
\end{aligned}
$$

by virtue of the hypothesis.

We further observe that

$$
\left|P_{1}(\omega)\right|^{r}=\left|\sum_{\mu_{q}<\omega}\left\{\left(\mu_{q} b_{q}\right)^{1 / r} \frac{A_{\lambda}^{k}\left(\omega-\mu_{q}\right)}{\left(\omega-\mu_{q}\right)}\right\} \times\left\{\left(\mu_{q} b_{q}\right)^{1 / r^{\prime}}\right\}\right|^{r} .
$$

Applying Hölder's inequality for sums with indices $r$ and $r^{\prime}$, we have 


$$
\begin{aligned}
\left|P_{1}(\omega)\right|^{r} & \leqq\left\{\sum_{\mu_{q}<\omega}\left|\mu_{q} b_{q}\right| \frac{\left|A_{\lambda}^{k}\left(\omega-\mu_{q}\right)\right|^{r}}{\left(\omega-\mu_{q}\right)^{r}}\right\}\left\{\sum_{\mu_{q}<\omega}\left|\mu_{q} b_{q}\right|\right\}^{r / r^{\prime}} \\
& =o\left(\omega^{r-1}\right) \sum_{\mu_{q}<\omega}\left|\mu_{q} b_{q}\right| \frac{\left|A_{\lambda}^{k}\left(\omega-\mu_{q}\right)\right|^{r}}{\left(\omega-\mu_{q}\right)^{r}}
\end{aligned}
$$

Hence, by using Theorem 1 of Srivastava [5] and the hypothesis of the theorem,

$$
\begin{aligned}
\int_{h}^{x}\left|P_{1}(\omega)\right|^{r} d \omega & \leqq \sum_{\mu_{q}<X}\left|\mu_{q} b_{q}\right| \int_{\mu_{q}}^{x} o\left(\omega^{r-1}\right) \frac{\left|A_{\lambda}^{k}\left(\omega-\mu_{q}\right)\right|^{r}}{\left(\omega-\mu_{q}\right)^{r}} d \omega \\
& \leqq o\left(X^{r-1}\right) \sum_{\mu_{q}<x}\left|\mu_{q} b_{q}\right| \int_{\mu_{q}}^{x} o(1)\left(\omega-\mu_{q}\right)^{r(k-1)} d \omega \\
& =o\left(X^{r-1}\right) \sum_{\mu_{q}<X}\left|\mu_{q} b_{q}\right| o\left(X^{r(k-1)+1}\right) \\
& =o\left(X^{r-1}\right) o\left(X^{r(k-1)+1}\right) \sum_{\mu_{q}<X}\left|\mu_{q} b_{q}\right| \\
& =o\left(X^{k r+1}\right),
\end{aligned}
$$

provided $k>1 / r^{\prime}$.

Similarly we can prove that

$$
\int_{h}^{x}\left|P_{2}(\omega)\right|^{r} d \omega=o\left(X^{k r+1}\right), \text { as } X \rightarrow \infty .
$$

Thus collecting our results, we have

$$
\int_{h}^{x}\left|\vec{C}_{p}^{k}(\omega)\right|^{r} d \omega=o\left(X^{k r+1}\right), \text { as } X \rightarrow \infty .
$$

This completes the proof of Theorem B.

6. Proof of Theorem A. The proof of this theorem follows immediately from Theorem B by omitting the last factors in (5.3) and (5.4).

\section{REFERENCES}

1. A. V. Boyd, Multiplication of strongly summable series, Proc. Glasgow Math. Assoc. 4 (1959/1960), 29-33.

2. A. V. Boyd and J. M. Hyslop, A definition for strong Rieszian summability and its relationship to strong Cesdro summability, Proc. Glasgow Math. Assoc. 1 (1952), 94-99. 
3. K. Chandrasekharan and S. Minakshisundaram, Typical means, Tata Institute of Fundamental Research Monograph, Oxford Univ. Press, New York, 1952.

4. T. M. Flett, Some remarks on strong summability, Quart. J. Math. Oxford Ser. (2) 10 (1959), 115-139.

5. Pramila Srivastava, On strong Rieszian summability of infinite series, Proc. Nat. Inst. Sci. India, Part A 23 (1957), 58-71.

GorakhPUR UNIVERSITy, GorakhPUR, INDIA

\section{ON HYPONORMAL OPERATORS}

\section{H. SHETH}

1. An operator $T$ defined on a Hilbert space $H$ is said to be hyponormal if $T^{*} T-T T^{*} \geqq 0$, or equivalently if $\left\|T^{*} x\right\| \leqq\|T x\|$ for every $x \in H$. An operator $T$ is said to be seminormal if either $T$ or $T^{*}$ is hyponormal. If $T$ is hyponormal, then $T-z I$ is also hyponormal for all complex values of $z$.

The spectrum of an operator $T$, in symbols $\sigma(T)$, is the set of all those complex numbers $z$ for which $T-z I$ is not invertible. A complex number $z$ is said to be an approximate proper value for the operator $T$ in case there exists a sequence $x_{n}$ such that $\left\|x_{n}\right\|=1$ and $\left\|(T-z I) x_{n}\right\|$ $\rightarrow 0$. The approximate point spectrum of an operator $T$, in symbols $\Pi(T)$, is the set of approximate proper values of $T$. The numerical range of an operator $T$, denoted by $W(T)$, is the set defined by the relation

$$
W(T)=\{(T x, x):\|x\|=1\} .
$$

$\mathrm{Cl}(W(T))$ will, as usual, denote the closure of $W(T)$. An operator $S$ is said to be similar to an operator $T$ in case there exists an invertible operator $A$ such that $S=A^{-1} T A$.

In this note, all the operators will relate to a Hilbert space $H$.

We shall prove the following theorem.

THEOREM. Let $N$ be a hyponormal operator. If for an arbitrary operator $A$, for which $0 \notin \mathrm{Cl}(W(A)), A N=N^{*} A$, then $N$ is self-adjoint.

Received by the editors April 11, 1966. 